\title{
Titanio: aspectos del material para uso en ortodoncia
}

\author{
Santiago Arango-Santander ${ }_{\star}, D_{D S}, B_{1}$, Carolina Ramírez-Vega, DDS 2 \\ Grupo gIOM \\ ${ }_{2}$ Especialización en Ortodoncia \\ Facultad de Odontología, Universidad Cooperativa de Colombia, Medellín, Colombia
}

Recibido: 15 de diciembre del 2014 Aprobado: 14 de marzo del 2015

*Autor de correspondencia: Santiago Arango Santander. Facultad de Odontología, Universidad Cooperativa de Colombia, sede Medellín. Carrera 47 n. 37 sur - 18, Medellín, Antioquia, Colombia. Teléfono: (57) 4 4446065. Correo electrónico: santiago.arango@campusucc.edu.co

Cómo citar este artículo: Arango-Santander S, Ramírez-Vega C. Titanio: aspectos del material para uso en ortodoncia. Rev Nac Odontol. 2016;12(23):63-71. doi:10.16925/od.v12i23.1423

Resumen. Introducción: el titanio y sus aleaciones han sido utilizados durante largo tiempo en Medicina y en Odontología debido a sus numerosas propiedades, entre las cuales están su alta resistencia a la corrosión y su gran biocompatibilidad. En el caso de la ortodoncia, este material y sus aleaciones tienen una historia relativamente corta, pero su uso se ha venido incrementando en los últimos años debido a sus excelentes propiedades mecánicas, como el efecto de memoria de forma y la superelasticidad, que hacen que sea un material con un extraordinario desempeño biomecánico, sobre todo en el caso de los alambres ortodóncicos, lo cual es altamente buscado en el área de la ortodoncia. Objetivo: examinar la historia del titanio en ortodoncia, sus principales características, sus propiedades físicas y mecánicas, y su uso dentro de la gama de materiales ortodóncicos disponibles en el mercado. Conclusión: en ortodoncia, el titanio es utilizado aleado con otros elementos (níquel, molibdeno y cobre, entre otros) que potencializan sus propiedades y permiten explotar sus cualidades clínicas.

Palabras clave: alambres ortodóncicos, aleaciones de titanio, efecto de memoria de forma, superelasticidad. 


\section{Titanium: Material Features for its Use in Orthodontics}

Abstract. Introduction: Titanium and its alloys have been long used in Medicine and Odontology due to their numerous properties, among them their high resistance to corrosion and outstanding biocompatibility. In the case of orthodontics, titanium and its alloys have a relatively short history, but its use has been increased during the last years, due to its excellent mechanical properties, like its design-in memory effect and super-elasticity, which provide this material with extraordinary biomechanical performance, especially in the case of orthodontic wire, which is highly searched for in the area of orthodontics. Objective: To examine the history of titanium in orthodontics, its main characteristics, its physical and mechanical properties and its use within a spectrum of orthodontic materials available in the market. Conclusion: Titanium alloys are used in conjunction with other elements (nickel, molybdenum and copper, amongst others) that leverage the titanium's properties and allow the operation of its clinical qualities.

Keywords: titanium alloys, orthodontic wires, design-in memory effect, super-elasticity.

\section{Titânio: aspectos do material para uso em ortodontia}

Resumo. Introdução: o titânio e suas ligações têm sido utilizadas durante muito tempo em Medicina e em Odontologia devido a suas numerosas propriedades, entre as quais sua alta resistência à corrosão e sua grande biocompatibilidade. No caso da ortodontia, este material e suas ligações possuem uma história relativamente breve, mas seu uso tem aumentado nos últimos anos, devido a suas excelentes propriedades mecânicas, como o efeito de memória de forma e a superelasticidade, que fazem com que seja um material com um extraordinário desempenho biomecânico, principalmente, no caso dos arames ortodônticos, sendo altamente buscado na área da ortodontia. Objetivo: revisar a história do titânio em ortodontia, suas principais características, propriedades físicas e mecânicas e seu uso dentro da gama de materiais ortodônticos disponíveis no mercado. Conclusão: em ortodontia, é utilizado o titânio ligado a outros elementos (níquel, molibdênio, cobre, entre outros), os quais potencializam as propriedades do titânio e permitem explorar suas qualidades clínicas.

Palavras chave: arames ortodônticos, ligações de titânio, efeito de memória de forma, superelasticidade. 


\section{Introducción}

El titanio es un elemento químico de símbolo $\mathrm{Ti}$ y numero atómico 22. Es un metal de transición no magnético de color gris plata; se considera que es el cuarto metal estructural más abundante en la superficie terrestre y el noveno en la gama de metales industriales, y no se encuentra en estado puro en la naturaleza, sino en forma de óxidos [1]. Su extracción es bastante simple, dado que no está a mucha profundidad en la corteza terrestre; sin embargo, su costoso procesamiento hace que sea considerado uno de los metales con mayor valor comercial [2].

Se puede hallar titanio en algunas rocas ígneas y sus sedimentos, en muchos minerales, sobre todo los que contienen hierro, y en organismos vegetales y animales. Los minerales más importantes de los que se extrae titanio son el rutilo (óxido de titanio) y la ilmenita, muy abundante en las arenas costeras [1]. Luego de su extracción, el titanio debe ser sometido a un proceso metalúrgico de refinado para prevenir su eventual reacción con sustancias gaseosas, tales como el nitrógeno, el oxígeno y el hidrógeno [1]. El titanio es liviano (43\% más que el acero), dúctil, fuerte y más rígido y resistente que el acero; además, puede ser fundido, colado y soldado [3].

Según la ASTM (American Society of Testing and Materials), la aleación más utilizada en el campo industrial es $3 \mathrm{Al}-2,5 \mathrm{~V}$ (94,5\% titanio, 3\% aluminio y $2,5 \%$ vanadio). En el campo médico - odontológico, las más usadas son Ti-6Al-4V y Ti-Al-Nb [2,4]. En ortodoncia, las aleaciones más comunes están formadas por níquel y titanio [4].

Aunque el titanio ha sido ampliamente usado en odontología y medicina, el objetivo de este artículo es discutir la historia del titanio en ortodoncia, sus principales características, propiedades físicas y mecánicas y su uso dentro de la gama de materiales ortodóncicos disponibles en el mercado, especialmente desde el punto de vista de los alambres.

\section{Historia}

El titanio fue descubierto en Inglaterra por William Gregor en 1791, pero poco después, en 1795, el químico alemán Martin H. Klaproth le dio el nombre de titanio (en honor a los titanes de la mitología griega) [5]. Sin embargo, el químico Matthew Hunter preparó por primera vez titanio metálico puro
$(99,9 \%)$ calentando tetracloruro de titanio $\left(\mathrm{TiCl}_{4}\right)$ con sodio a $700-800^{\circ} \mathrm{C}$ en un reactor de acero [1].

En 1946, William J. Kroll desarrolló un método para producirlo comercialmente mediante la reducción del $\mathrm{TiCl}_{4}$ con magnesio. En este proceso de cambio cristalográfico, el titanio pasa de su fase alfa (estructura de cuerpo centrado o hexagonal compacta) a una fase beta (disposición de cuerpo centrado o cúbica tetragonal) a $883^{\circ} \mathrm{C}$ con un punto de fusión de $1680^{\circ} \mathrm{C}$ [2]. El titanio se fusiona al vacío bajo gases inertes (por ejemplo, con el argón) para prevenir la oxidación, lo que puede volverlo frágil y disminuir su ductilidad $[1,2]$.

Con respecto a la historia de las aleaciones utilizadas en ortodoncia, en 1962 Buehler descubrió el Nitinol (lo llamó así haciendo referencia a sus siglas en inglés "Nickel- Titanium Naval Ordnance Laboratory"). En 1970, Andreasen introdujo en la ortodoncia la combinación intermetálica 50/50 (en porcentaje) de níquel y titanio en la Universidad de Iowa [6]. En 1974, la Unitek Corporation patentó una aleación martensítica estabilizada bajo el nombre de Nitinol $^{\mathrm{Ts}}$. En 1977, la fase beta del titanio fue estabilizada a temperatura ambiente y, así, se produjo la aleación titanio-molibdeno. En 1986, se presentaron dos aleaciones superelásticas: el NiTi Japonés y el NiTi Chino. En 1990, salió al mercado el Neosentalloy como una aleación martensítica verdadera. En 1994, se presentaron tres aleaciones cobre-níquel-titanio con un efecto de memoria de forma a los $27^{\circ} \mathrm{C}, 35^{\circ} \mathrm{Co} 40^{\circ} \mathrm{C}$. Más recientemente, arcos libres de níquel o de titanio-niobio han sido usados como arcos de finalización [7].

\section{Propiedades biológicas}

Pasivación: el titanio, como la mayoría de los metales, se oxida si se expone a la atmósfera [8]. Entre sus óxidos están el $\mathrm{TiO}, \mathrm{TiO}_{2}$ y el $\mathrm{Ti}_{2} \mathrm{O}_{3} \mathrm{El}$ más usado por su estabilidad es el $\mathrm{TiO}_{2}$. En un milisegundo, se forma una capa de $10 \AA^{2}$ (1 nanómetro) de óxido que en un minuto se convertirá en una capa de $100 \AA$ (10 nanómetros) cuando el metal es expuesto a un ambiente con oxígeno. Este fenómeno se conoce como "pasivación natural" del titanio. La capa pasivada no sufre ruptura o daño bajo condiciones fisiológicas, aunque esta pasivación natural puede ser reforzada con un baño de ácido nítrico sobre el metal [1]. Esta capa de óxido ofrece una superficie biocompatible, resistente a 
la corrosión y es altamente resistente a la fractura durante los cambios de fuerzas aplicadas y provee un buen medio en las mecánicas ortodóncicas de deslizamiento [2, 9-12].

Sin embargo, el hecho de que el titanio sufra pasivación no significa que no se pueda corroer [1]. Es importante tener en cuenta que la presencia de sustancias como el flúor (el cual produce liberación de iones del titanio) en productos para la higiene oral, debe ser tenida en cuenta cuando se evalúa la efectividad de la relación arco-bracket, pues se ha observado cierto grado de aumento en la corrosión del titanio que incrementa la fricción entre el arco y la ranura del bracket, lo cual puede disminuir la efectividad del comportamiento del material; e incluso generar fractura [3, 10,13-22]. La liberación de iones en $\mathrm{pH}$ ácido $(\geq 3,75)$ ha sido prácticamente indetectable, lo que sugiere una excelente protección contra la corrosión por parte de la capa pasiva [11, 16, 22-25]. Finalmente, se ha observado corrosión en casos en que el titano tiene una alta absorción de hidrógeno debido a un aumento exagerado en la tensión que produce una disminución de la ductilidad y de la resistencia a la tracción $[22,26]$. Sin embargo, en condiciones normales, el titanio es un metal que posee gran resistencia a la corrosión en la cavidad oral [27].

Biocompatibilidad y citotoxicidad: en cuanto a la biocompatibilidad y el nivel de citotoxicidad, el dióxido de titanio ha mostrado un promedio de disolución muy bajo en estado pasivado [1]. El nivel normal de presencia de titanio en tejidos humanos es de $50 \mathrm{ppm}$. Sin embargo, se han observado niveles entre 100 a $300 \mathrm{ppm}$ alrededor de implantes de titanio que no han representado riesgo humano. De hecho, la mayoría de las investigaciones in vivo e in vitro ha demostrado ausencia de citotoxicidad con el uso de aleaciones de titanio [1, 9, 23, 28-31] aunque algunos autores han reportado casos aislados de hipersensibilidad a componentes de las aleaciones, como es el caso específico del níquel [30].

\section{Estructura}

El titanio es un material alotrópico que existe en dos formas: una estructura hexagonal compacta $(\alpha-\mathrm{Ti})$ hasta los $882^{\circ} \mathrm{C}$ y una estructura cúbica centrada en el cuerpo $(\beta-\mathrm{Ti})$ por encima de esta temperatura La aleación alfa tiene una microestructura de fase sencilla, mientras que la fase beta tiene una estructura de dos fases [32]. El titanio puede ser aleado con elementos como aluminio, vanadio o molibdeno, entre otros, sin perder su capacidad de pasivación $[1,8]$. El aluminio es el estabilizador de la fase alfa y sirve para aumentar la resistencia, disminuir el peso de la aleación y aumentar la temperatura de transformación de la fase alfa a la beta. El vanadio o el molibdeno son estabilizadores de la fase beta, lo que causa que esta fase se mantenga a una temperatura inferior a la temperatura de transformación. El estabilizador beta ofrece resistencia a la corrosión $[1,32]$.

\section{Usos en ortodoncia}

Brackets: el titanio ha venido incursionando en una amplia gama de materiales usados en ortodoncia. Las primeras aleaciones se desarrollaron para fabricar alambres de diferentes calibres; luego se usaron en ciertos aditamentos como resortes de NiTi, minitornillos para anclaje ortodóncicos [33] y brackets de titanio comercialmente puro [34], debido a los reportes de los profesionales y a los estudios in vitro respecto a casos de alergia al níquel contenido en las aleaciones de acero y a su corrosión. [35,36].

En cuanto a los brackets de titanio, es bien sabido que durante la mecánica de deslizamiento, la resistencia a la fricción es un factor importante para el movimiento de los dientes y debe ser controlada durante el tratamiento para tener una aplicación de fuerzas óptimas. Se sabe que a mayor resistencia a la fricción será necesario aumentar la magnitud de las fuerzas de ortodoncia para superar dicha resistencia. En este sentido, se ha encontrado que los brackets de titanio desarrollan menos fuerza friccional a medida que el tamaño de los arcos aumenta [37] y que tienen una mejor estabilidad dimensional y estructural [38] comparados con los brackets de acero.

Alambres: como ya se mencionó antes, el uso del titanio y las aleaciones de titanio en medicina y odontología ha aumentado durante los últimos años [1,12]. Las aleaciones más importantes de uso ortodóncico para la fabricación de alambres se conocen como NiTi (níquel- titanio), $\beta$ - titanio o TMA (titanio-molibdeno), CuNiTi (cobre-níquel-titanio), entre otras $[4,12]$.

Aleaciones de níquel-titanio (NiTi): existen dos fases grandes en este tipo de aleaciones. La fase austenítica tiene una estructura cúbica centrada en el cuerpo y ordenada que ocurre a alta temperatura 
y bajo esfuerzo. En esta fase, la aleación es más rígida [39]. Cuando la aleación es enfriada a través del rango de transformación por temperatura, ocurren cambios grandes en el límite elástico, rigidez y resistencia eléctrica. El cambio que se presenta en la estructura cristalina, conocido como transformación martensítica, tiene como consecuencia un cambio en las propiedades físicas de la aleación [40]. La fase martensítica tiene una estructura hexagonal, monoclínica o triclínica desordenada que ocurre a baja temperatura y alto esfuerzo [4, 41-43]. En la transformación no se observan cambios macroscópicos, excepto si se aplica una fuerza externa debido a que la aleación es más dúctil en la fase martensítica [40]. Las transformaciones martensíticas y austeníticas tienen temperaturas iniciales (As, Ms) y temperaturas finales (Af, Ms) [39]. Si la fase prevalente es "activa" (llamada así cuando una fase se transforma en la otra mediante activación térmica o mecánica) o "estable", dependerá de la historia de la deformación que ha sufrido la aleación [44]. Las aleaciones de NiTi tienen propiedades específicas como el efecto de memoria de forma (efecto termoelástico) y el efecto de superelasticidad (conocido como pseudoelasticidad).

La superelasticidad (llamada pseudoelasticidad en ciencia de materiales) es el fenómeno mediante el cual es posible inducir transformaciones martensíticas localizadas por medio de deflexiones, independientemente de la temperatura, a una aleación en fase austenítica $[4,12,43]$. Este efecto es el análogo mecánico del efecto de memoria de forma.

La memoria de forma es la capacidad de una aleación de recuperar su forma inicial luego de ser deformada y ser sometida a temperaturas superiores a su temperatura final austenítica (Af) $[4,12]$, es decir, es la capacidad del material para volver a su forma original de fabricación (fase austenítica) después de haber sido deformado en la fase martensítica. Estas transiciones de fase pueden ocurrir solo a través de cambios en la temperatura [43]. Este efecto está asociado con una transformación austenita-martensita reversible que ocurre rápidamente debido a un emparejamiento cristalográfico a nivel atómico [4]. En las aleaciones donde la temperatura de transformación austenítica está cerca, pero por debajo de la temperatura de la cavidad oral, una transformación martensítica puede ser inducida por una deformación grande [12, 42]. Las aplicaciones clínicas en ortodoncia de este efecto son notorias, ya que a través de los ciclos de temperatura y la deflexión, el alambre en la fase austenítica es capaz de "memorizar" una forma, incluyendo una forma de arco, que es útil en ortodoncia. Al disminuir la temperatura, la aleación pasa a fase martensítica, donde es más flexible y se deforma fácilmente [39].

Los alambres ortodóncicos de níquel-titanio pueden ser alternativamente clasificados en tres categorías de acuerdo con su comportamiento [6]:

- Aleación martensítica estabilizada: no tiene efecto de memoria de forma ni superelasticidad, ya que en el proceso de fabricación se crea una estructura martensítica estable.

- Aleación martensítica activa: emplea la termoelasticidad para lograr efecto de memoria de forma.

- Aleación austenítica activa: sufre una transformación martensítica inducida por esfuerzo (comportamiento superelástico).

Nitinol: la aleación original fue desarrollada a principios de la década de los sesenta como parte de un programa para fabricar un material que fuera no magnético, resistente a la sal y a prueba de agua. Esta aleación fue llamada Nitinol. Es una forma martensítica estabilizada en la que el endurecimiento por trabajo suprime la fase de transformación. Esta aleación original no tiene memoria de forma ni superelasticidad, aunque sí posee un bajo módulo de elasticidad y un rango de trabajo amplio que lo hacen útil cuando son necesarias deflexiones considerables del material $[4,39]$.

En ortodoncia, esta aleación fue introducida con la combinación inter-metálica 50/50 (en porcentaje) de níquel y titanio [6]. Actualmente, la aleación de este tipo más común es el Nitinol 55, compuesto por $55 \%$ níquel y $45 \%$ titanio (por peso). El Nitinol 55 tiene un alto grado de memoria mecánica a la temperatura ambiente, pero no acepta el tratamiento térmico $[3,9,40,45]$.

En diversos estudios, el Nitinol ha mostrado que la rugosidad de la superficie aumenta durante el uso clínico, lo que incrementa a su vez la fricción con otros materiales, siendo esto de importancia clínica según el tipo de biomecánica que se use en un tratamiento ortodóncico [9, 11, 13, 15, 24, 46, 47].

NiTi Japonés: en 1978, la empresa Furukawa Electric Company Ltda. produjo un alambre austenítico activo conocido como NiTi Japonés cuyas propiedades principales son una excelente recuperación elástica y superelasticidad [40]. Es ampliamente 
usado para arcos de ortodoncia, ya que produce movimientos rápidos sin deformación permanente del arco [48, 49]. Gracias a la superelasticidad, los arcos de esta aleación entran fácilmente en la ranura del bracket y no causan inconformidad o malestar al paciente [49].

NiTi Chino: esta aleación austenítica activa fue desarrollada especialmente para usos en ortodoncia por el doctor Tien Hua Cheng, en el Instituto General de Investigación para metales no ferrosos en Pekín, China. El NiTi Chino tiene una recuperación elástica que es 4,4 veces mayor que la del acero inoxidable y es 1,6 veces mayor que la del Nitinol [50]. La deformación del NiTi Chino no depende del tiempo y, a diferencia del Nitinol, este no continúa deformándose en cantidades importantes en boca cuando se hacen ajustes o activaciones. La rigidez característica es determinada por la cantidad de activación. El índice de carga-deformación en pequeñas activaciones es considerablemente superior al índice en las activaciones de gran tamaño. Esta aleación de NiTi es muy conveniente si se requiere baja rigidez y gran deflexión. Su mayor rigidez en activaciones pequeñas hace que sea más eficaz que las aleaciones tradicionales, en las cuales el nivel de fuerza puede ser muy bajo. Sus características de poco endurecimiento durante el trabajo, temperatura de transición mucho más baja y que tenga una fase austenita con buen rendimiento de las propiedades mecánicas hacen que difiera significativamente del Nitinol convencional [50].

Neosentalloy (Neo superelastic nickel titanium alloy): en 1990, aparece por primera vez esta aleación martensítica activa (efecto de memoria de forma) con la cual era posible utilizar un arco rectangular de alambre como arco inicial. El profesional puede elegir un arco que genere 100, 200 o 300 gramos de fuerza [51]. Esta aleación tiene una estructura completamente austenítica final cerca de la temperatura de la cavidad oral $\left(32,7^{\circ} \mathrm{C}\right)$. Ofrece una fuerza casi constante a lo largo de la fase de carga y descarga durante el uso de un alambre de esta aleación [4], es decir, posee una histéresis considerable para el rango de transición de temperatura en ambas direcciones para la transformación completa (martensita a austenita) [51]. Además, tiene una excelente resistencia a la corrosión en comparación con otras aleaciones NiTi [52].

Aleación cobre-níquel-titanio: esta aleación se ha introducido con el objetivo de aumentar la resistencia a la fatiga y el rango de histéresis térmica. Se ha observado que la presencia de cobre en un rango de 5-10\% cambia la estructura cristalográfica de la fase de baja temperatura. De hecho, la histéresis térmica hace que el rango de la formación entre austenita (por calentamiento) y martensita (por enfriamiento) sea más cercano que el de las aleaciones de NiTi convencionales, lo que significa que la adición de cobre a la aleación aumenta el rango de temperatura de transición alrededor del nivel de la temperatura intraoral, por lo cual permite un control más definido del efecto de memoria de forma. Dicho de otro modo, la activación y desactivación del arco puede realizarse mediante el consumo de bebidas y alimentos fríos o calientes [53]. El alambre conocido como Copper-NiTi (Ormco Corporation, Glendora, Estados Unidos) se fabrica en tres variantes según la temperatura austenita final (Af): $27^{\circ} \mathrm{C}, 35^{\circ} \mathrm{C}$ y $40^{\circ} \mathrm{C}$. El alambre de $27^{\circ} \mathrm{C}$ mostrará el efecto de memoria de forma a la temperatura oral. El de $35^{\circ} \mathrm{C}$ no mostrará este efecto con tanta frecuencia en la cavidad oral debido a que la temperatura final austenítica está muy cercana a la temperatura de la cavidad oral. El alambre de $40^{\circ} \mathrm{C}$ solo podrá ser activado mediante el consumo de alimentos calientes porque la temperatura austenítica final está por encima de la temperatura oral [53].

Se ha visto que la adición de cobre a las aleaciones puede afectar la biocompatibilidad, aunque ha sido un tema controvertido, dado que algunos autores no han encontrado dicha afectación con la presencia de cobre [54]. La adición de cobre disminuye la susceptibilidad de las aleaciones de níquel-titanio a la corrosión por picadura (pitting corrosion) [19].

Aleación titanio-molibdeno: esta aleación, usada en ortodoncia, es fabricada por la casa comercial Ormco Corporation (Glendora, Estados Unidos) y es distribuida bajo el nombre comercial tMa (Titanium-Mollybdenum Alloy) [4]. Esta aleación beta titanio posee un excelente balance de propiedades, incluyendo alta elasticidad, baja rigidez, alta capacidad de ser formado y capacidad de soldadura directa [4]. Sin embargo, un inconveniente importante es su alto coeficiente de fricción [55].

Se considera que las aleaciones beta titanio tienen un alto potencial de uso en ortodoncia debido a que la máxima deflexión elástica de un aparato ortodóncico aumenta con la resistencia a la fluencia y la relación del módulo de elasticidad del material y el beta titanio es una de las más altas, dado 
que puede obtener una gran resistencia a la fluencia mientras mantiene un mínimo módulo de elasticidad. Debido a la predominancia de la estructura cúbica centrada en el cuerpo en la fase beta, esta aleación tiene buena capacidad de ser formada aun después de un considerable trabajo en frío y posee buena estabilidad en medio ambiente [3, 45, 56-58].

Aleación de titanio libre de níquel: Esta aleación ha sido desarrollada debido a la alta toxicidad encontrada en algunas aleaciones, como la $\mathrm{Ti}$ $6 \mathrm{Al}$ [30]. Entre estas aleaciones tóxicas está el titanio-niobio ( Ti $6 \mathrm{Al} 7 \mathrm{Nb}$ ) y otras como Ti $15 \mathrm{Al} 2.5 \mathrm{Fe}$, Ti $15 \mathrm{Sn} 4 \mathrm{Nb} 2 \mathrm{Ta} 0.2 \mathrm{Pd}$ y Ti $15 \mathrm{Zr} 4 \mathrm{Nb} 4 \mathrm{Ta} 0.2 \mathrm{Pd}$ que no son usadas en el campo de la ortodoncia [59].

La rigidez del titanio-niobio en el doblez es casi la mitad de la del acero inoxidable, mientras que en torsión es más o menos un tercio. Estas características le permiten al clínico utilizar el titanio-niobio para dobleces sin niveles de fuerza excesiva. El efecto de retorno a su punto original en los dobleces es $14 \%$ más bajo que el del acero, mientras que en torsión es un poco más alto, lo cual posibilita que se utilice el alambre hasta para correcciones mayores de tercer orden [60]. La tabla 1 es un resumen de las propiedades más relevantes de las distintas aleaciones utilizadas para fabricar alambres de ortodoncia.

Tabla 1. Propiedades de las aleaciones utilizadas en ortodoncia

\begin{tabular}{|l|l|}
\hline \multicolumn{1}{|c|}{ Aleación } & \multicolumn{1}{c|}{ Propiedades } \\
\hline Ni-Ti & Súper elasticidad y memoria de forma \\
\hline Nitinol & $\begin{array}{l}\text { Bajo módulo de elasticidad, amplio rango de } \\
\text { trabajo y alta fricción }\end{array}$ \\
\hline Ni-Ti Japonés & $\begin{array}{l}\text { Excelente recuperación elástica y súper elasti- } \\
\text { cidad }\end{array}$ \\
\hline Ni-Ti Chino & $\begin{array}{l}\text { Baja rigidez, gran deflexión y bajo endureci- } \\
\text { miento por trabajo }\end{array}$ \\
\hline Neosentalloy & $\begin{array}{l}\text { Memoria de forma y alta resistencia a la } \\
\text { corrosión }\end{array}$ \\
\hline Cu-Ni-Ti & $\begin{array}{l}\text { Memoria de forma y baja susceptibilidad a la } \\
\text { corrosión }\end{array}$ \\
\hline Ti-Mo & $\begin{array}{l}\text { Alta elasticidad, baja rigidez, alta capacidad de } \\
\text { ser formado, capacidad de soldadura directa y } \\
\text { alto coeficiente de fricción }\end{array}$ \\
\hline
\end{tabular}

Fuente: elaboración propia

\section{Conclusiones}

El titanio es un material que ha logrado gran aceptación dentro del campo de la medicina y la odontología, especialmente en las áreas de la implantología y la ortodoncia, gracias a su alta biocompatibilidad con los tejidos orales y su adaptación a las condiciones particulares de un tratamiento, como sucede en el caso de la ortodoncia.

En ortodoncia, el titanio es utilizado aleado con otros elementos (níquel, molibdeno, cobre, entre otros), los cuales potencializan sus propiedades y permiten explotar sus cualidades clínicas. Entre las propiedades más importantes que tienen las aleaciones de titanio están la superelasticidad y la termoelasticidad, las cuales se observan en las aleaciones níquel-titanio; la gran resistencia a la fatiga es una característica importante de la aleación con cobre; la excelente resistencia a la fluencia con un mínimo módulo de elasticidad es observada en la aleación con molibdeno; y la alta biocompatibilidad con buena soldabilidad está presente en la aleación con niobio.

Es esencial que el ortodoncista conozca el comportamiento y las propiedades de cada una de las aleaciones a fin de elegir la mejor opción a la hora de usar algún tipo de aditamento en los tratamientos ortodóncicos y, así, lograr los objetivos deseados desde el punto de vista biomecánico.

\section{Referencias}

[1] Parr GR, Gardner LK, Toth RW. Titanium: the mystery metal of implant dentistry. Dental materials aspects. J Prosthet Dent. 1985 Sep;54(3):410-4.

[2] Affairs ACoS. Titanium applications in dentistry. J Am Dent Assoc. 2003 Mar;134(3):347-9.

[3] Burstone CJ, Goldberg AJ. Beta titanium: a new orthodontic alloy. Am J Orthod. 1980 Feb;77(2):121-32.

[4] Brantley WA, Eliades T. Orthodontic materials. Scientific and clinical aspects. Stuttgart: Thieme; 2001. 310 p.

[5] Burke R. Hazardous materials chemistry for emergency responders. 2a ed. Boca Ratón: CRC Press; 2002.

[6] Kusy RP. A review of contemporary archwires: their properties and characteristics. Angle Orthod. 1997;67(3):197-207. 
[7] Kusy RP. Orthodontic biomaterials: from the past to the present. Angle Orthod. 2002 Dec;72(6):501-12.

[8] Steinemann SG. Titanium--the material of choice? Periodontol 2000. 1998 Jun;17:7-21.

[9] Huang HH. Variation in corrosion resistance of nickel-titanium wires from different manufacturers. Angle Orthod. 2005 Jul;75(4):661-5.

[10] Kusy RP, O'Grady P W. Evaluation of titanium brackets for orthodontic treatment: Part II--The active configuration. Am J Orthod Dentofacial Orthop. 2000 Dec;118(6):675-84.

[11] Kusy RP, Whitley JQ, Ambrose WW, Newman JG. Evaluation of titanium brackets for orthodontic treatment: part I. The passive configuration. Am J Orthod Dentofacial Orthop. 1998 Nov;114(5):558-72.

[12] Soto E. ¿Cómo seleccionar aleaciones de niquel-titanio? Punto de Contacto. 2002;7(1):10-4.

[13] Chern Lin JH, Lo SJ, Ju CP. Biocorrosion study of titanium-nickel alloys. J Oral Rehabil. 1996 Feb;23(2):129-34.

[14] Cioffi M, Gilliland D, Ceccone G, Chiesa R, Cigada A. Electrochemical release testing of nickel-titanium orthodontic wires in artificial saliva using thin layer activation. Acta Biomater. 2005 Nov;1(6):717-24.

[15] Clocheret K, Willems G, Carels C, Celis JP. Dynamic frictional behaviour of orthodontic archwires and brackets. Eur J Orthod. 2004 Apr;26(2):163-70.

[16] Huang HH, Chiu YH, Lee TH, Wu SC, Yang HW, Su $\mathrm{KH}$, et al. Ion release from NiTi orthodontic wires in artificial saliva with various acidities. Biomaterials. 2003 Sep;24(20):3585-92.

[17] Kaneko K, Yokoyama K, Moriyama K, Asaoka K, Sakai J, Nagumo M. Delayed fracture of beta titanium orthodontic wire in fluoride aqueous solutions. Biomaterials. 2003 May;24(12):2113-20.

[18] Li X, Wang J, Han EH, Ke W. Influence of fluoride and chloride on corrosion behavior of NiTi orthodontic wires. Acta Biomater. 2007 Sep;3(5):807-15.

[19] Pun DK, Berzins DW. Corrosion behavior of shape memory, superelastic, and nonsuperelastic nickel-titanium-based orthodontic wires at various temperatures. Dent Mater. 2008 Feb;24(2):221-7.

[20] Schiff N, Boinet M, Morgon L, Lissac M, Dalard F, Grosgogeat B. Galvanic corrosion between orthodontic wires and brackets in fluoride mouthwashes. Eur J Orthod. 2006 Jun;28(3):298-304.

[21] Schiff N, Grosgogeat B, Lissac M, Dalard F. Influence of fluoride content and $\mathrm{pH}$ on the corrosion resistance of titanium and its alloys. Biomaterials. 2002 May;23(9):1995-2002.

[22] Yokoyama K, Hamada K, Moriyama K, Asaoka $\mathrm{K}$. Degradation and fracture of $\mathrm{Ni}-\mathrm{Ti}$ supere- lastic wire in an oral cavity. Biomaterials. 2001 Aug;22(16):2257-62.

[23] Eliades T. Orthodontic materials research and applications: part 2. Current status and projected future developments in materials and biocompatibility. Am J Orthod Dentofacial Orthop. 2007 Feb;131(2):253-62.

[24] Huang HH. Variation in surface topography of different $\mathrm{NiTi}$ orthodontic archwires in various commercial fluoride-containing environments. Dent Mater. 2007 Jan;23(1):24-33.

[25] Wang J, Li N, Rao G, Han EH, Ke W. Stress corrosion cracking of NiTi in artificial saliva. Dent Mater. 2007 Feb;23(2):133-7.

[26] Yokoyama K, Kaneko K, Ogawa T, Moriyama K, Asaoka K, Sakai J. Hydrogen embrittlement of work-hardened $\mathrm{Ni}$-Ti alloy in fluoride solutions. Biomaterials. 2005 Jan;26(1):101-8.

[27] Grimsdottir MR, Hensten-Pettersen A. Surface analysis of nickel-titanium archwire used in vivo. Dent Mater. 1997 May;13(3):163-7.

[28] Eliades T, Pratsinis H, Kletsas D, Eliades G, Makou M. Characterization and cytotoxicity of ions released from stainless steel and nickel-titanium orthodontic alloys. Am J Orthod Dentofacial Orthop 2004 Jan;125(1):24-9.

[29] Kapanen A, Ilvesaro J, Danilov A, Ryhanen J, Lehenkari P, Tuukkanen J. Behaviour of nitinol in osteoblast-like Ros-17 cell cultures. Biomaterials. 2002 Feb;23(3):645-50.

[30] Kolokitha OE, Chatzistavrou E. A severe reaction to ni-containing orthodontic appliances. Angle Orthod. 2009 Jan;79(1):186-92.

[31] Mockers O, Deroze D, Camps J. Cytotoxicity of orthodontic bands, brackets and archwires in vitro. Dent Mater. 2002 Jun;18(4):311-7.

[32] Park J, Bronzino J. Biomaterials: principles and applications. 2 ed. Tehran: Amirkabir University of Technology (AUT) Press Center; 2003.

[33] Morais LS, Serra GG, Muller CA, Andrade LR, Palermo EF, Elias CN, et al. Titanium alloy mini-implants for orthodontic anchorage: immediate loading and metal ion release. Acta Biomater. 2007 May;3(3):331-9.

[34] Gioka C, Bourauel C, Zinelis S, Eliades T, Silikas N, Eliades G. Titanium orthodontic brackets: structure, composition, hardness and ionic release. Dent Mater. 2004 Sep;20(7):693-700.

[35] Noble J, Ahing SI, Karaiskos NE, Wiltshire WA. Nickel allergy and orthodontics, a review and report of two cases. Br Dent J. 2008 Mar 22;204(6):297-300.

[36] Sfondrini MF, Cacciafesta V, Maffia E, Scribante A, Alberti G, Biesuz R, et al. Nickel release from new 
conventional stainless steel, recycled, and nickel-free orthodontic brackets: An in vitro study. Am J Orthod Dentofacial Orthop. 2010 Jun;137(6):809-15.

[37] Kapur R, Sinha PK, Nanda RS. Comparison of frictional resistance in titanium and stainless steel brackets. Am J Orthod Dentofacial Orthop. 1999 Sep;116(3):271-4.

[38] Kapur R, Sinha PK, Nanda RS. Comparison of load transmission and bracket deformation between titanium and stainless steel brackets. Am J Orthod Dentofacial Orthop. 1999 Sep;116(3):275-8.

[39] Santoro M, Nicolay OF, Cangialosi TJ. Pseudoelasticity and thermoelasticity of nickel-titanium alloys: a clinically oriented review. Part I: Temperature transitional ranges. Am J Orthod Dentofacial Orthop. 2001 Jun;119(6):587-93.

[40] Thompson SA. An overview of nickel-titanium alloys used in dentistry. Int Endod J. 2000 Jul;33(4):297-310.

[41] Han S, Quick DC. Nickel-titanium spring properties in a simulated oral environment. Angle Orthod. 1993 Spring;63(1):67-72.

[42] Kusy RP. Nitinol alloys: so, who's on first? Am J Orthod Dentofacial Orthop. 1991 Sep;100(3):25A-6A.

[43] Maganzini AL, Wong AM, Ahmed MK. Forces of various nickel titanium closed coil springs. Angle Orthod. 2010 Jan;80(1):182-7.

[44] Kusy RP, Wilson TW. Dynamic mechanical properties of straight titanium alloy arch wires. Dent Mater. 1990 Oct;6(4):228-36.

[45] Goldberg J, Burstone CJ. An evaluation of beta titanium alloys for use in orthodontic appliances. J Dent Res. 1979 Feb;58(2):593-99.

[46] Kusy RP, Whitley JQ, Mayhew MJ, Buckthal JE. Surface roughness of orthodontic archwires via laser spectroscopy. Angle Orthod. 1988 Jan;58(1):33-45.

[47] Wichelhaus A, Geserick M, Hibst R, Sander FG. The effect of surface treatment and clinical use on friction in NiTi orthodontic wires. Dent Mater. 2005 Oct;21(10):938-45.

[48] Hudgins JJ, Bagby MD, Erickson LC. The effect of long-term deflection on permanent deformation of nickel-titanium archwires. Angle Orthod. 1990 Winter;60(4):283-8.

[49] Miura F, Mogi M, Ohura Y, Hamanaka H. The super-elastic property of the Japanese NiTi alloy wire for use in orthodontics. Am J Orthod Dentofacial Orthop. 1986 Jul;90(1):1-10.

[50] Burstone CJ, Qin B, Morton JY. Chinese NiTi wire--a new orthodontic alloy. Am J Orthod. 1985 Jun;87(6):445-52.

[51] Teramoto A. Sentalloy: The Story of Superelasticity. Mexico City: Universidad Tecnológica de México; 2010. Available from: https://www.dentsply.com/ content/dam/dentsply/master/document/W/WHITE-PAPER---SENTALLOY---The-story-of-Superelasticity-by-Dr-Alberto-Teramoto-lnbffjq-en-1509.pdf

[52] Es-Souni M, Fischer-Brandies H. On the properties of two binary NiTi shape memory alloys. Effects of surface finish on the corrosion behaviour and in vitro biocompatibility. Biomaterials. 2002 Jul;23(14):2887-94.

[53] Brantley WA, Guo W, Clark WA, Iijima M. Microstructural studies of 35 degrees $\mathrm{C}$ copper $\mathrm{Ni}-\mathrm{Ti}$ orthodontic wire and TEM confirmation of low-temperature martensite transformation. Dent Mater. 2008 Feb;24(2):204-10.

[54] Es-Souni M, Brandies HF. On the transformation behaviour, mechanical properties and biocompatibility of two niti-based shape memory alloys: NiTi42 and NiTi42Cu7. Biomaterials. 2001 Aug;22(15):2153-61.

[55] Verstrynge A, Van Humbeeck J, Willems G. In-vitro evaluation of the material characteristics of stainless steel and beta-titanium orthodontic wires. Am J Orthod Dentofacial Orthop. 2006 Oct;130(4):460-70.

[56] Johnson E. Relative stiffness of beta titanium archwires. Angle Orthod. 2003 Jun;73(3):259-69.

[57] Kusy RP. Comparison of nickel-titanium and beta titanium wire sizes to conventional orthodontic arch wire materials. Am J Orthod. 1981 Jun;79(6):625-9.

[58] Lim Y, Quick A, Swain M, Herbison P. Temperature effects on the forces, moments and moment to force ratio of nickel-titanium and TMA symmetrical T-loops. Angle Orthod. 2008 Nov;78(6):1035-42.

[59] Niinomi N. Mechanical properties of biomedical titanium alloys. Materials Science and Engineering A. 1998;243:231-6.

[60] Dalstra M, Denes G, Melsen B. Titanium-niobium, a new finishing wire alloy. Clin Orthod Res. 2000 Feb;3(1):6-14. 